\title{
ASTROMETRY OF LARGE SKY SURVEYS
}

\author{
W.F. van ALTENA, T.M. GIRARD, I. PLATAIS
}

\begin{abstract}
One of the major uses of the Large Sky Surveys such as the Palomar and SERC Schmidt Surveys is the derivation of positions for faint objects discovered in the course of other surveys. The HST Guide Star Catalogue has proved to be of great help in deriving positions for such objects, but the well-known, and unavoidable, systematic errors in the GSC positions limit the accuracy of the final positions. In this Review, we will describe techniques that can be used to successfully transfer a reference frame from the bright FK5 stars to the small confines of a CCD chip containing only faint objects with the goal of maintaining an accuracy of approximately 0.1 arcsecond in the final positions.
\end{abstract}

